\title{
Effect of Different POSS Structures on Thermal and Morphological Properties of a Biodegradable Polyester ${ }^{\dagger}$
}

\author{
Adriana N. Frone *, Ioana Chiulan, Madalina Oprea, Alexandru S. Stoian, Denis M. Panaitescu, \\ Augusta R. Gabor and Cristian A. Nicolae

\begin{abstract}
National Institute for Research \& Development in Chemistry and Petrochemistry ICECHIM,Polymer Department, 202 Splaiul Independentei, 060021 Bucharest, Romania; ioana.chiulan@icechim.ro (I.C.); madalinna.calarasu@gmail.com (M.O.); stoian.sergiu@gmail.com (A.S.S.); panaitescu@icechim.ro (D.M.P.); ralucagabor@yahoo.com (A.R.G.) ca_nicolae@yahoo.com (C.A.N.)

* Correspondence: adriana.frone@icechim.ro

+ Presented at the 15th International Symposium "Priorities of Chemistry for a Sustainable Development" PRIOCHEM, Bucharest, Romania, 30th October-1st November 2019.
\end{abstract}

Published: 16 October 2019

Keywords: poly(lactic acid); silica based nanofillers; thermal properties; atomic force microscopy

The quest for materials with properties similar to those of engineering plastics but derived from renewable resources remains a continuous need of our time. Poly(lactic acid) (PLA) is a linear aliphatic thermoplastic polyester, intensively studied and currently used for various applications because of its biodegradability and availability on the market at a price close to polypropylene [1]. However, its slow crystallization rate, low thermal stability, and excessive brittleness are disadvantages that limit the wider applicability of PLA [2]. Extensive research has showed that an effective way to improve PLA properties is the addition of nanofillers. Because of their nanoscale dimensions, biocompatibility, recyclability, nonflammability, nonabrasive, and nonmigrating features, polyhedral oligomeric silsesquioxanes (POSS) could be an interesting choice as fillers for PLA [3]. The aim of this study was to evaluate the POSS influence on the thermal, morphological, and mechanical behavior of a poly(l-lactic acid) matrix (PLLA). POSS fillers (trisilanol-isooctyl polyhedral oligomeric silsesquioxanes-TSio-POSS, allyl-heptaisobutyl-ALib-POSS, and aminopropyl-heptaisobutyl polyhedral oligomeric silsesquioxane-APib-POSS) (Sigma-Aldrich) were incorporated into a PLLA matrix (4043D, NatureWorks Ingeo) by a melt compounding method. The morphological, thermal, mechanical, and surface properties of PLLA/POSS nanocomposites were evaluated by atomic force microscopy (AFM), thermogravimetric analysis (TGA), differential scanning calorimetry (DSC), dynamic mechanical analysis (DMA), and water contact angle measurements (CA). AFM analysis showed a more organized structure for the TSio-POSS nanocomposite, whereas some aggregates were detected mainly on the surface of the PLLA/ALibPOSS film. CA results indicated that POSS improved the hydrophobicity of the PLLA matrix, with the exception of the nanocomposite containing APib-POSS - probably due to the better incorporation of APib-POSS in the PLLA matrix, as also shown by AFM. The addition of POSS improved the thermal stability of the PLLA matrix, as demonstrated from the characteristic temperatures of the TG/DTG curves. A slight shift of $T_{m}$ nanocomposites towards lower values was observed as a result of the plasticizing effect of POSS and good miscibility between PLLA and POSS.The downward shift of $T_{c c}$ indicated that ALib and APib-POSS nanoparticles enhanced the nucleating activity and the rate of crystallization of the PLLA, which was also confirmed by the higher degree of crystallinity values. Incorporation of POSS led to a decrease of the storage modulus and increased PLLA flexibility, which was noticed in the glassy state, bellow $T_{g}$; beyond the $T_{g}$ region, all the PLLA/POSS nanocomposites displayed higher storage modulus values as compared with neat PLLA. The loss modulus curves 
indicated that POSS addition decreases the melt viscosity of the corresponding PLLA nanocomposites. The self-assembled POSS molecules were well dispersed in the PLLA matrix, as shown by AFM images. Furthermore, an increase in thermal stability was noticed, and both $T_{\text {on }}$ and $T_{5 \%}$ were modified, depending on the POSS type. Except for TSio-POSS, the fillers acted as plasticizers, lowering the $\mathrm{E}^{\prime}$ of the PLLA matrix in the glassy region and increasing the $\mathrm{E}^{\prime \prime}$ above the glass transition temperature $\left(T_{g}\right)$.

Acknowledgments: This work was supported by a grant of theMinistry of Research and Innovation, CNCSUEFISCDI, PN-III-P1-1.1-TE-2016-2164, No. 94/2018, Biocompatible multilayer polymer membranes with tuned mechanical and antiadherent properties (BIOMULTIPOL), within PNCDI III and Contract 23N/2019, PN 1923020104 .

\section{References}

1. Dolores, F.M.; Jesus, F.M.; Cobos, M. Effect of polyhedral oligomeric silsesquioxane (POSS) derivative on the morphology, thermal, mechanical and surface properties of poly(lactic acid)-based nanocomposites. J. Mater. Sci. 2016, 51, 3628-3642.

2. Huang, L.; Tan, J.; Li, W.; Zhou, L.; Liu, Z.; Luo, B.; Lu, L.; Zhou, C. Functional polyhedral oligomeric silsesquioxane reinforced poly(lactic acid) nanocomposites for biomedical applications. J. Mech. Behav. Biomed. Mater. 2019, 90, 604-614.

(C) 2019 by the authors. Licensee MDPI, Basel, Switzerland. This article is an open access article distributed under the terms and conditions of the Creative Commons Attribution (CC BY) license (http://creativecommons.org/licenses/by/4.0/). 